\title{
Funcionamiento y Estabilidad en el Tiempo de un Sistema Energético con Batería PEM a Combustible y sus Componentes Periféricos
}

\author{
Juan I. Franco ${ }^{(1,2)}$, Alfredo R. Sanguinetti ${ }^{(1)}$, Gabino E. Colángelo ${ }^{(3)}$ y Héctor J. Fasoli ${ }^{(1) *}$ \\ (1) Instituto de Enseñanza Superior del Ejército, Escuela Superior Técnica "Grl Manuel N Savio", \\ Cabildo 15, 1426, Buenos Aires-Argentina (e-mail: hfasoli@citefa.gov.ar). \\ (2) Instituto de Investigaciones Científicas y Técnicas para la Defensa (CITEDEF), \\ San Juan B. de La Salle 4397, 1603, Villa Martelli, provincia de Buenos Aires-Argentina \\ (e-mail: jfranco@citefa.gov.ar). \\ (3) Facultad de Ingeniería, Universidad de Buenos Aires, Av. Paseo Colón 850, 1063, \\ Buenos Aires-Argentina \\ * autor a quien debe ser dirigida la correspondencia.
}

Recibido Sept. 15, 2009; Aceptado Nov. 20, 2009; Versión Final recibida Ene. 03, 2010

Resumen

En este trabajo se presenta el desarrollo completo de una batería de tipo membrana de electrolito polimérico (proton exchange membrane fuel cell stack, PEMFCS), y se muestra el funcionamiento de un sistema experimental completo que está siendo probado en la Base Esperanza que el Ejército Argentino posee en la Antártida. El conjunto desarrollado permite trabajar con un mínimo de componentes periféricos y ser manejado por personal entrenado con capacitación básica para su operación y mantenimiento. La resistencia óhmica de la batería no difiere de la que se obtendría con seis elementos en serie; su pendiente de Tafel es de $80 \mathrm{mV} /$ déc. Las pruebas de estabilidad en el tiempo alcanzaron 2278 horas y la energía entregada en ese tiempo fue de $4.2 \mathrm{kWh}$.

Palabras clave: pilas a combustible, batería de pilas PEM, membrana de electrolito polimérico, resistencia óhmica

\section{Work Conditions and Time Stability Study on a Fuel Cell Stack and its Peripheral Components}

\begin{abstract}
The development and working performance of a proton exchange membrane fuel cell stack (PEMFCS) which was installed at Base Esperanza belonging to the Argentinean Army in the Antarctic territory is presented. The set designed and installed allows working with minimum peripherical devices and can be operated and maintained by personnel with basic training. The ohmic resistance of the battery is the same that could be obtained from six elements in series; the Tafel slope is 80 $\mathrm{mV} / \mathrm{dec}$. Stability experiments were performed during 2278 hours, and the energy generated was 4.2 kWh.
\end{abstract}

Keywords: fuel cells, PEM fuel cell stack, proton exchange membrane, ohmic resistance 


\section{INTRODUCCIÓN}

Las pilas y baterías a combustible que se consideran más promisorias para funcionar a baja temperatura están basadas en electrolito polimérico: a las ventajas tecnológicas relacionadas específicamente con este electrolito debe sumarse la ventaja general de las pilas a hidrógeno que provocan muy baja o nula contaminación ambiental durante su funcionamiento (Kordesh y Simader, 1996). Sin embargo, la optimización de la energía que puede obtenerse de estos sistemas depende del control de una serie de variables tales como temperatura (Park y Li, 2006), flujo de los gases reaccionantes (Dohle et al, 2003), estequiometria, presión y humedad (Jiao y Zhou, 2008; Andreaus y Scherer, 2003; Hyum y Kim, 2004), todos los cuales influyen también sobre el envejecimiento del electrolito (Knights et al., 2003). La optimización se logra usualmente adicionando al dispositivo electroquímico un conjunto de equipos cuyo gasto energético debe restarse de la energía producida. Por otra parte, la utilización del sistema por el público no especializado debe ser lo más simple posible si deseamos que en el futuro esta tecnología sea utilizada en la vida cotidiana; el trabajo de Rodatz et al. (2003) puede considerarse uno de los más representativos en ese sentido ya que analiza bajo condiciones prácticas los aspectos operativos de baterías de electrolito polimérico (PEM).

El desarrollo y parámetros de funcionamiento de baterías ("stack") de pilas a combustible con membrana de electrolito polimérico (PEM, por "polymer electrolyte membrane") fue presentado por nosotros recientemente (Franco et al., 2007). En este trabajo se presenta el desarrollo completo de una batería a la que denominamos EA-5, que superó ampliamente los requisitos establecidos por el convenio firmado a fines de 2004 por el Ejército Argentino con las empresas ENARSA y Aeropuertos Argentina 2000 (potencia máxima prevista 5 W, para mayo 2007) (Fasoli et al., 2008).

Se muestra asimismo el funcionamiento de un sistema experimental completo que fue probado en la Base Esperanza del Ejército Argentino en la Antártida. Éste fue construido deliberadamente simple de manera de disminuir los gastos energéticos de componentes periféricos, aun a pesar de que la batería no trabaje en condiciones de rendimiento óptimo. Esto facilitó la operación del conjunto por parte de personal no especializado, con un mínimo de entrenamiento en su operación y mantenimiento, lo que constituye un indicio de la dirección que puede seguirse al desarrollar sistemas energéticos autónomos para puntos aislados (Fasoli y Franco, 2004) y es, a nuestro entender, la primera referencia de un sistema de estas características construido con el concepto de "conecte y use" ("plug and play"). Al mismo tiempo, la experiencia sirve para probar el comportamiento de la batería durante períodos de funcionamiento prolongado y también bajo ciclos de reposo de duración variable, dependientes de las circunstancias que se dan en la Base; la respuesta a este trato es indicación de la robustez del sistema electroquímico.

El conjunto puede representarse mediante la secuencia (1), donde FC corresponde a la batería de pilas a combustible (del inglés para Fuel Cell) y TV se refiere a una carga que en este caso está representada por un televisor portátil.

$\stackrel{220 V A C}{\longrightarrow}$ Fuente. $($ VCC $) \rightarrow$ Electrolizador $\stackrel{\mathrm{H}_{2}+\mathrm{O}_{2}}{\longrightarrow} \mathrm{FC} \rightarrow \mathrm{TV}$

\section{METODOLOGÍA}

El punto de partida para la obtención del conjunto membrana-electrodo (EMA) fue anticipado anteriormente (Casanova et al., 2003) y ha sido motivo de la presentación de una patente (Sanguinetti et al., 2007). Para la preparación de los EMA se emplea membrana de Nafion ${ }^{\circledR} 117$ y catalizador de platino en soporte grafito de Alfa Aesar; los reactivos químicos son de calidad para análisis (Merck o Aldrich); detalles sobre las características de estos electrodos se discuten en Franco et al. (2007): la concentración de platino en el EMA es $1,0 \mathrm{mg} / \mathrm{cm}^{2}$ y la superficie activa es 16 $\mathrm{cm}^{2}$.

La Figura 1 muestra una fotografía de la batería, a la que denominamos EA-5. Las curvas de descarga se obtuvieron con un sistema de carga electrónica Agilent $3301 \mathrm{~A}$. 
El electrolizador utilizado para alimentar la batería también fue diseñado y construido en nuestro laboratorio. Consta de un cilindro de acrílico con cierre hermético y electrodos de acero inoxidable. El electrolito empleado es solución acuosa de $\mathrm{KOH} 30 \%$. La separación de los gases se logra de manera sencilla mediante un cilindro de acrílico interpuesto entre ambos electrodos. El análisis del hidrógeno que se produce en el cátodo indica que bajo las condiciones de trabajo la concentración de oxígeno en el hidrógeno es menor que $0,1 \%$ v/v (sensor CiTicel $\mathrm{C} / \mathrm{NHL}$ ). Los gases generados por el electrolizador se desprenden a una presión apenas mayor que la atmosférica, controlada por un sistema eudiométrico que se emplea para lavar los gases, garantizar $100 \%$ de humedad relativa en ambos y que simultáneamente sirve como válvula para la alimentación de la batería. El electrolizador se conecta a una fuente de CC marca Kepco Power Supply Modelo MBT (0-55 V, 0-7 A), la que a su vez toma corriente eléctrica $(220 \mathrm{~V}, 50 \mathrm{~Hz})$ producida mediante un sistema que emplea un generador eólico instalado en la Base. Detalle del conjunto formado por el electrolizador y los cilindros lavadores se observa en la fotografía de la Figura 2.

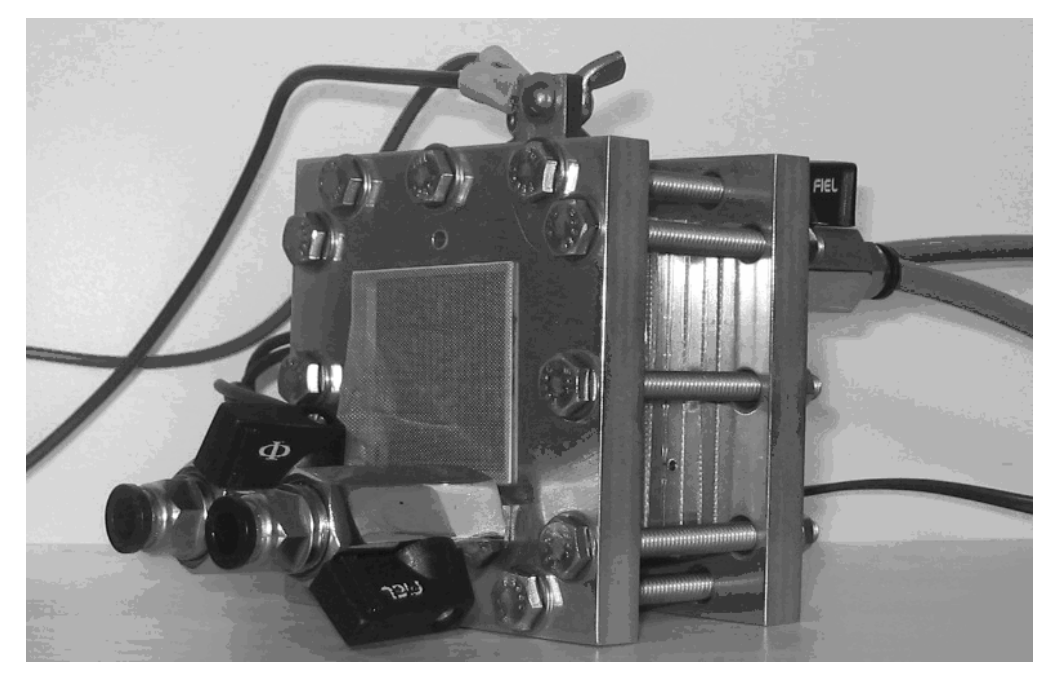

Fig. 1: Vista del "stack" EA-5, parte principal del sistema instalado en la Base Esperanza.

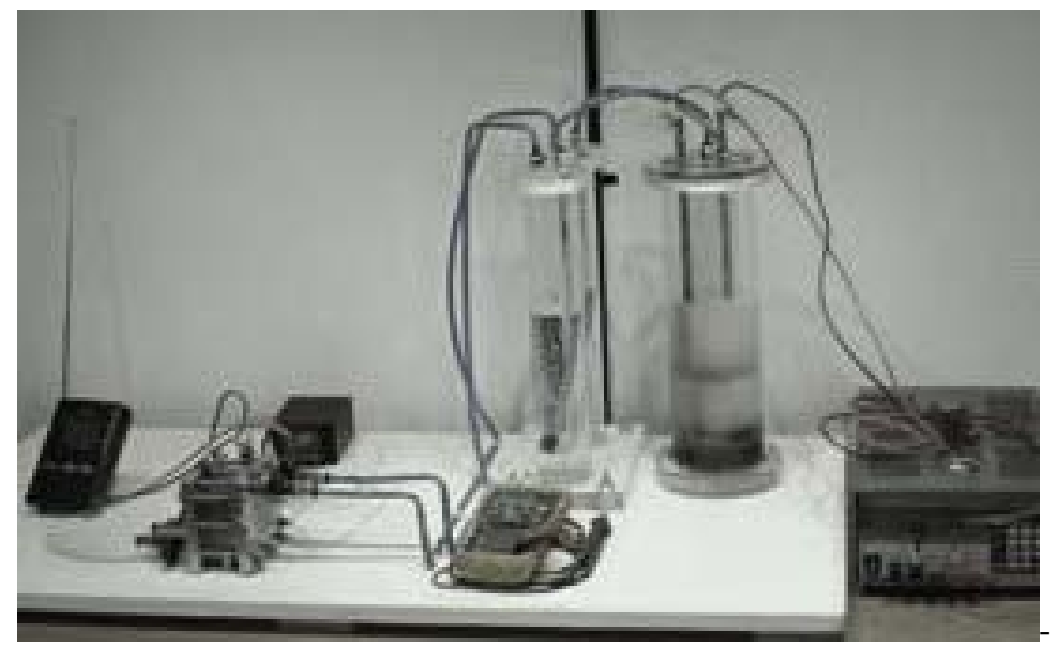

Fig. 2: Sistema instalado en la Base Esperanza. A la izquierda, un televisor portátil conectado a la batería EA-5.

Un televisor portátil marca CITIZEN modelo STO551R (6 V CC, 2,5 W) se emplea como carga de la batería. El "stack" está provisto de dos resistencias tipo "estampilla" adheridas a las placas terminales de aluminio anodizado con el propósito de calentar la batería para llevarla a la temperatura deseada. Un sistema idéntico al descripto funciona también en nuestro laboratorio de Villa Martelli (provincia de Buenos Aires) con el propósito de estudiar simultáneamente su comportamiento e introducirle mejoras. 
Durante los períodos de funcionamiento continuo las mediciones de potencial se realizan a cualquier hora del día mediante un multímetro Fluke 189 contrastado contra otro similar existente en nuestro laboratorio.

El conjunto se trasladó a la Base Esperanza durante la misión del rompehielos Almirante Irízar del verano 2006-2007. La instalación se realizó durante la Navidad de 2006 y la descarga se inició el 3 de enero de 2007 y continuó luego con intermitencias debido a situaciones imprevistas dentro de la Base, tales como el accidente del rompehielos Almirante Irízar y el incendio de una escuela, hechos que obligaron a atender con poca regularidad el funcionamiento del dispositivo. Desde aquella fecha se realizaron medidas de tensión a diferentes horas del día y a diferentes temperaturas; en estos períodos las únicas paradas fueron para restituir agua al electrolizador o para purgar a la batería de la presencia del agua acumulada. La atención esporádica permitió comprobar la respuesta a períodos más o menos prolongados de reposo y la rapidez de llegada a régimen de descarga. Los datos obtenidos son enviados quincenalmente a nuestro laboratorio de Villa Martelli para ser analizados y procesados.

\section{RESULTADOS Y DISCUSIÓN}

\section{Características y parámetros de funcionamiento}

En la Figura 3 se representa gráficamente la potencia entregada por la batería EA-5 en función de la corriente para las siguientes condiciones de trabajo (Franco et al., 2007):
Tensión a circuito abierto, $\mathrm{V}_{\mathrm{oc}}: 6,0 \mathrm{~V}$
Potencia máx., $\mathrm{P}_{\text {máx: }}: 12,5 \mathrm{~W}$ (extrapolado)
Densidad de corriente a $\mathrm{P}_{\text {máx }}: 5,5 \mathrm{~A}$
Temperatura: $25^{\circ} \mathrm{C}$
Presión parcial de $\mathrm{H}_{2}, \mathrm{P}_{\mathrm{H}}: 736,3 \mathrm{mmHg}$
Humedad relativa del hidrógeno: 100\%
Presión parcial de $\mathrm{O}_{2}, \mathrm{P}_{\mathrm{O}}: 736,3 \mathrm{mmHg}$
Humedad relativa del oxígeno: $100 \%$
Flujo de hidrógeno, $\phi_{H}: 133,0 \mathrm{~mL}(\mathrm{CNPT}) / \mathrm{min}$
Flujo de oxígeno, $\phi_{\circ}: 66,5 \mathrm{~mL}(\mathrm{CNPT}) / \mathrm{min}$

Las condiciones fueron elegidas de manera de facilitar la operación del sistema por personal con adiestramiento mínimo. En general, los sistemas PEM son diseñados para funcionar bajo diferentes condiciones de temperatura (Jiao y Zhou, 2008); en nuestro caso la temperatura de operación es el resultado de utilizar el burbujeo de los gases en agua como humidificador a temperatura ambiente (18 a $25^{\circ} \mathrm{C}$ ). Por otra parte, la presión parcial del agua tiene efectos significativos sobre el funcionamiento de las pilas tipo PEM (Qi y Kaufman, 2002). El empleo de humedad relativa $100 \%$ tiende a independizar el comportamiento del sistema frente a los cambios de temperatura ambiente y, por lo tanto, de la presión de vapor de agua.

En la Figura 3 se representa también la diferencia de potencial entre bornes de la batería ("stack") en función de la corriente de la batería. Este "stack" está formado por 6 elementos en serie: si la batería estuviera funcionando idealmente esta curva debería ser la resultante de la suma de cada uno de los elementos. En la Figura 4 se realiza la misma representación que en la Figura 3 pero la diferencia de potencial se ha dividido por seis y se ha superpuesto además una descarga típica de una pila de un solo elemento obtenido con la misma tecnología (pila CITEST-5); en este caso en el eje horizontal se expresa la densidad de corriente $\mathbf{j}\left(\mathrm{en} \mathrm{mA} \mathrm{cm}^{-2}\right.$ ) y en el eje vertical secundario la densidad de potencia (en $\mathrm{mW} \mathrm{cm}^{-2}$ ) Puede observarse que la superposición de ambas curvas $\mathrm{V}$-j es evidente y significativa, indicando que en el "stack" cada elemento se comporta casi como si fuera independiente de los otros. La curva de densidad de potencia en función de la densidad de corriente por elemento del "stack" también coincide con la de la pila elemental lo que indica que, dentro de los errores experimentales del método, no hay pérdida de potencia al pasar de la pila a la batería por conexión de los elementos individuales formados por placas bipolares. 
Un análisis más completo y que permite llegar a la misma conclusión puede hacerse considerando el cálculo de la resistencia de polarización del sistema. La resistencia de polarización superficial $r_{p}$ está definida por:

$r_{p}=-\left(\frac{\partial E_{i}}{\partial j}\right)_{\text {Est.estacionario }}$

donde Ei es el potencial eléctrico y $j$ la densidad de corriente en estado estacionario.

Esta resistencia superficial $\left(\Omega . \mathrm{cm}^{2}\right)$ (Casanova et al., 2003), puede obtenerse de derivar la ecuación (3), utilizada habitualmente para la representación del potencial de la pila E como función de la densidad de corriente j (Ticianelli et al., 1998; Antolini et al., 2002).

$E=E^{\circ}-b \log j-r . j$

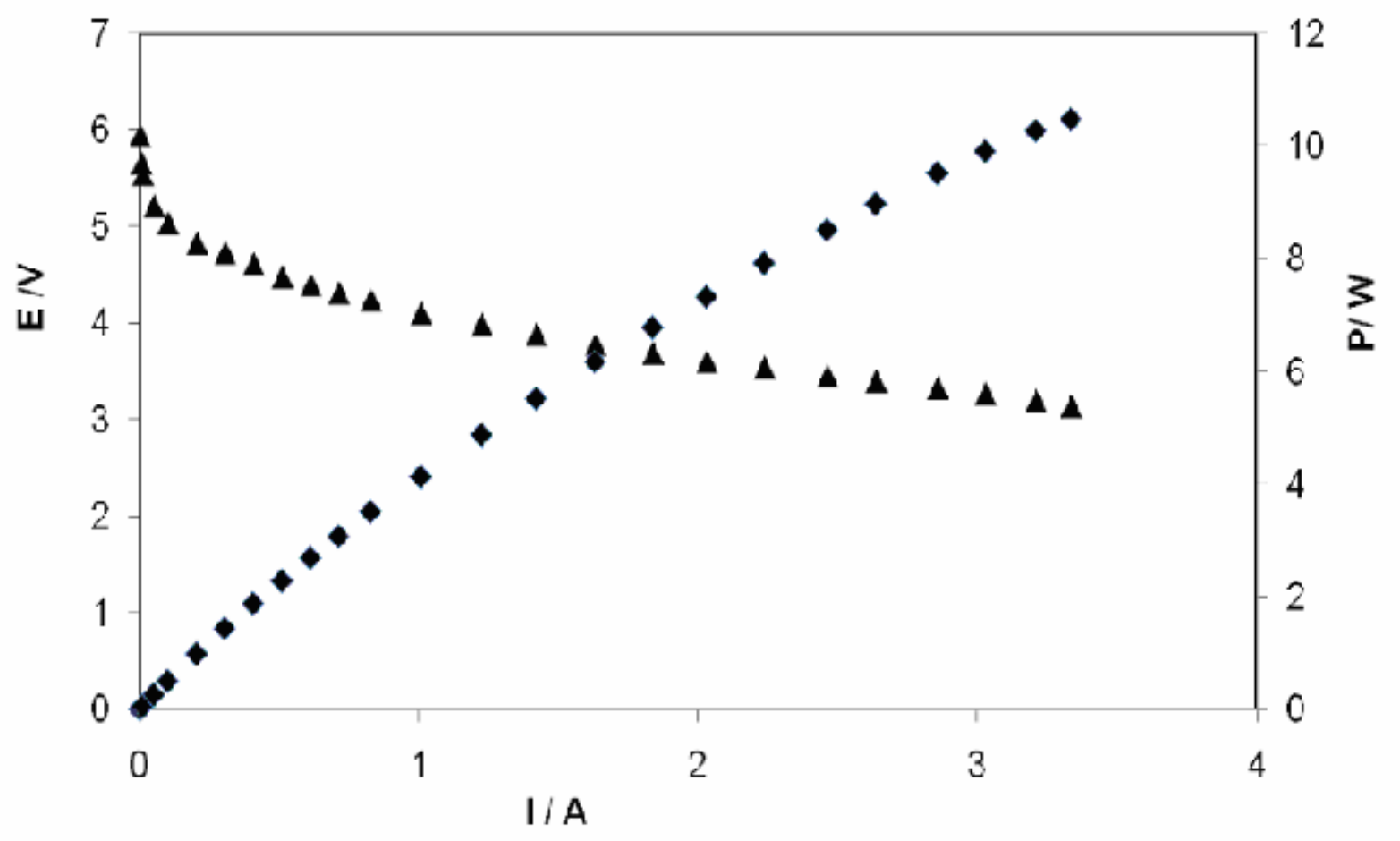

Fig.3: Curva V-I ( $\mathbf{\Delta})$ y de potencia ( $\mathbf{a})$ para el "stack" descripto en este trabajo.

En (3), $E_{r}$ es el potencial reversible de la pila y $E^{\circ}=E_{r}+b \log j_{o}$, donde $j_{o} y$ b son los parámetros de Tafel para la reacción de reducción del oxígeno y $r$ representa la resistencia superficial, la cual es suma de las resistencias por unidad de área de los fenómenos de transferencia de carga del oxígeno y del hidrógeno, la caída óhmica del electrolito y los electrodos y de las contribuciones de las limitaciones de transporte de masa de los gases. La densidad de corriente es j, siendo $\mathrm{j}=\mathrm{I} / \mathrm{S}$, donde I es la intensidad de corriente (en ampere, A) y $S$ la superficie de los electrodos $\left(\mathrm{en} \mathrm{cm}^{2}\right.$ ). Entonces, derivando la ecuación (3) respecto a la densidad de corriente j, resulta la resistencia de polarización superficial $r_{p}\left(\Omega . \mathrm{cm}^{2}\right)$. Si $r_{p}$ se divide por la superficie de los electrodos se obtiene:

$R_{p}=B / I+R$

donde $R_{p}(\Omega)$ es la resistencia de polarización, I $(A)$ es la corriente, $B(B=b / 2,303)$ es una constante proporcional a la suma de los parámetros $b$ de Tafel de las reacciones de transferencia de carga del oxígeno y del hidrógeno y $R(\Omega)=r S$. 


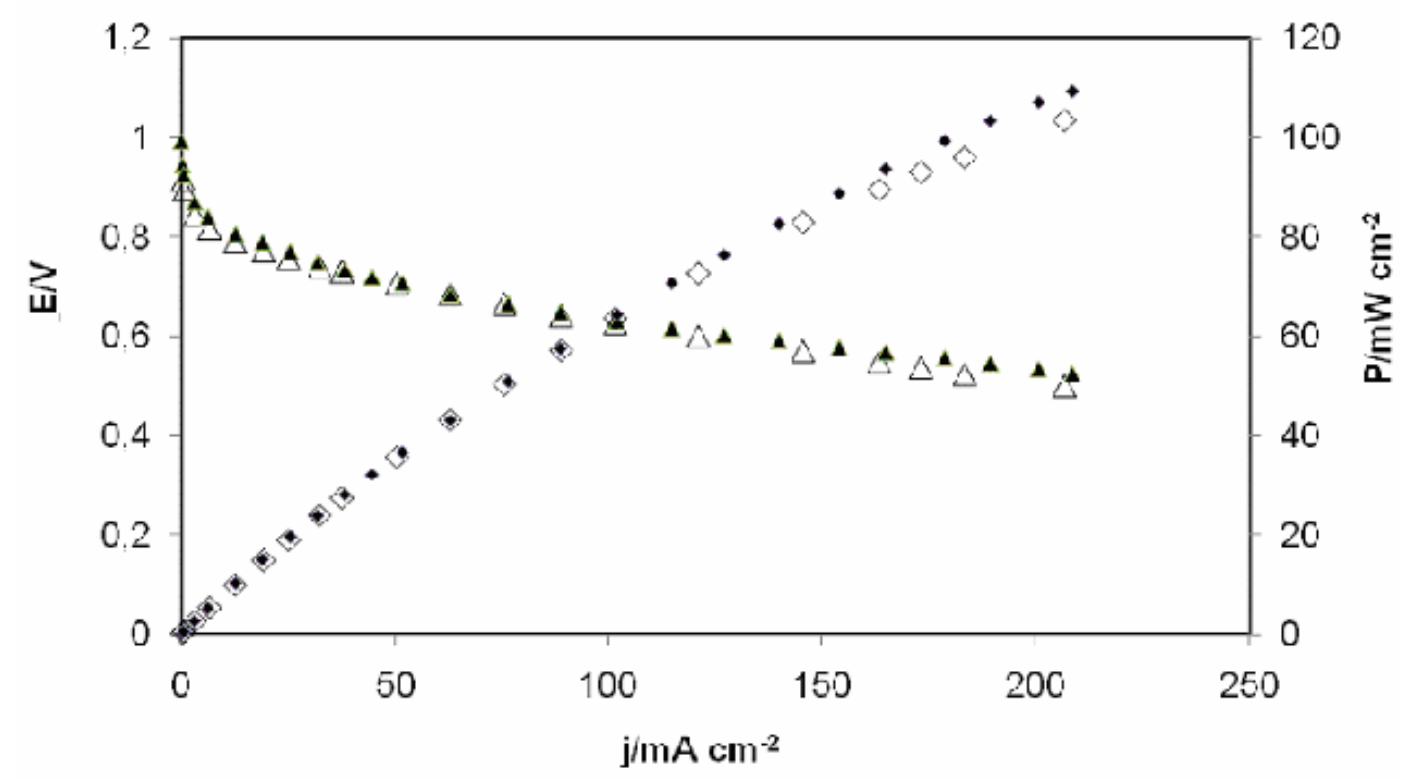

Fig. 4: Comparación de la curva de desempeño por elemento del "stack" EA-5 (E/6, puntos marcados con $\boldsymbol{\Delta}$ y $\mathbf{m}$ ) con la de la pila CITEST-5 de un solo elemento (curvas con $\Delta \mathrm{y} \square$ ).

En la Figura 5 se observa la dependencia de la resistencia de polarización del "stack" dividida por seis en función de la intensidad de corriente; en la misma figura se representa la resistencia de polarización de la pila de un único elemento. Dada la superposición casi perfecta de ambas curvas se concluye que el "stack" no agrega elementos resistivos adicionales (dentro de los límites de error de la experiencia) a la impedancia de la pila elemental. La resistencia de polarización se obtuvo de la derivación numérica de la curva experimental de polarización. La utilización de la ecuación (4) conduce a valores de b para el elemento simple de $75 \mathrm{mV} /$ década y en el caso de la batería de 80 $\mathrm{mV} /$ década a densidades de corriente bajas y medias.

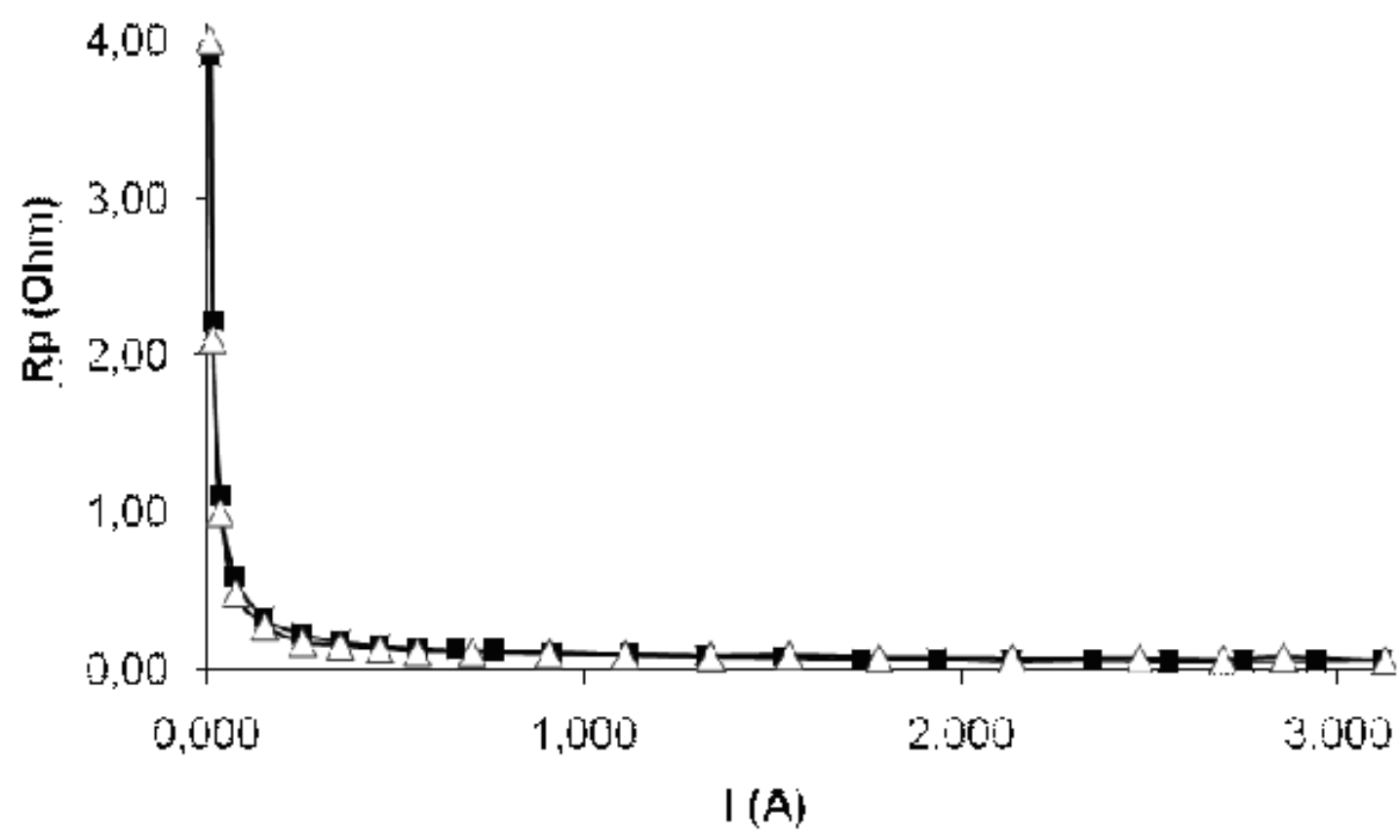

Fig. 5: Resistencia de polarización $\left(R_{p}\right)$ vs intensidad de corriente (I). Se representan en forma conjunta la resistencia de polarización de la pila CITEST-5 (curva con $\Delta$ ) y la del "stack" EA-5 dividida por 6 (घ). 
Los valores del coeficiente $b$ de la ecuación de Tafel han sido informados por varios autores como comprendidos entre 57 y $126 \mathrm{mV} /$ década; así, Passos y Ticianelli (2002) dan valores teóricos para la pendiente de Tafel (b) para la reacción de reducción del oxígeno de $70 \mathrm{mV} /$ década para potenciales $\mathrm{E}>0,85 \mathrm{~V}$ y de $140 \mathrm{mV} /$ década a $\mathrm{E}<0,85 \mathrm{~V}$. Estos autores utilizan un valor medio de 120 $\mathrm{mV} /$ década a $85^{\circ} \mathrm{C}$ para b. Por otro lado, Zhang et al. (2001) obtienen, a temperatura ambiente (313-343 K) sobre Nafion/Pt,C, pendientes de Tafel para la reacción de reducción del oxígeno entre 70-72,3 mV/década y 117,1-125,6 mV/década para $\mathrm{E}>0,85 \mathrm{~V}$ y $\mathrm{E}<0,85 \mathrm{~V}$, respectivamente.

Utilizando impedancia compleja sobre una pila PEM para medir la resistencia de polarización, V.A.Paganin et al. (1998) obtuvieron valores comprendidos entre 68 y $100 \mathrm{mV} /$ década para E> 0,85. Giddey et al .(2004) obtienen a partir de curvas de polarización de baterías formadas por 2, 4, 8 y 15 elementos que operan con hidrógeno-aire valores promedios del coeficiente $b$ comprendidos entre 122,7 y $85,9 \mathrm{mV} /$ década a $60^{\circ} \mathrm{C}$. Después de varios ciclos de descarga obtienen valores de $b$ comprendidos entre 58,2 y $69,1 \mathrm{mV} /$ década. Los procesos irreversibles que generan la polarización de los electrodos pueden expresarse con $R_{p}$, tal como se explicó más arriba. Esta $R_{p}$ produce un efecto similar el efecto Joule cuando la intensidad de corriente I circula por el "stack", con lo que $R_{p}$ permite calcular el autocalentamiento de la batería producido por la existencia de los fenómenos irreversibles.

\section{Comportamiento en el tiempo}

La estabilidad a través del tiempo constituye un aspecto clave para la evaluación de este tipo de dispositivos. Como se dijo, las descargas se iniciaron el 3 de enero de 2007. El equipo estuvo en funcionamiento desde entonces hasta mayo de 2008, cuando regresó a Buenos Aires para su inspección; la batería cumplió un total de 2278 horas de trabajo bajo carga. En la Figura 6 se representa gráficamente la diferencia de potencial de la batería en carga expresada en voltio en función del tiempo expresado en hora. Se han utilizado diferentes marcadores para indicar la temperatura del sistema, la que se mide en forma continua: se observa una buena estabilidad del potencial con el tiempo, con ligeros cambios esperables por el consumo variable del televisor.

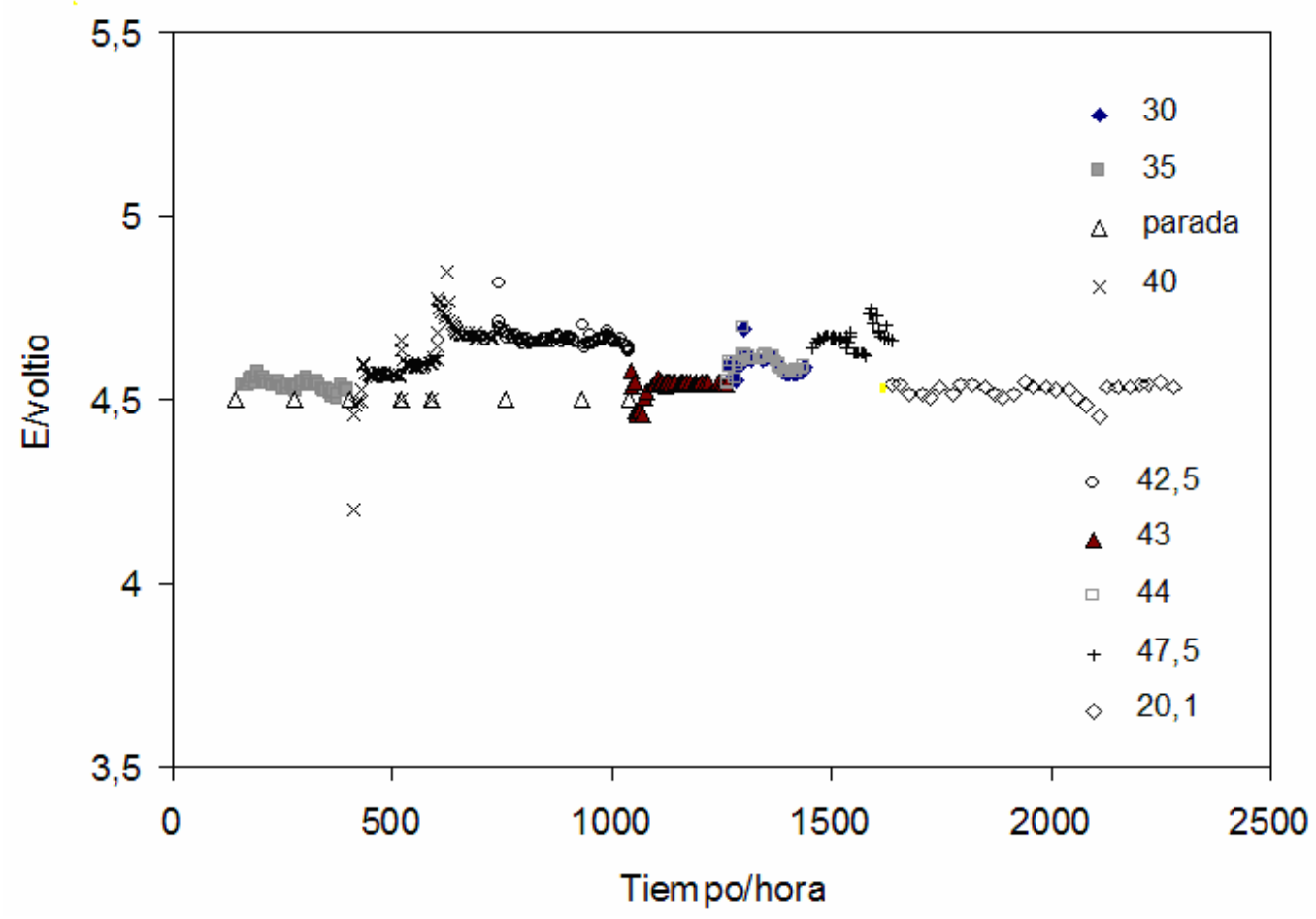

Fig. 6: Representación de la diferencia de potencial "vs." tiempo: 2278 horas de funcionamiento del sistema con el "stack" EA-5, a diferentes temperaturas: El recuadro de la derecha da las referencias para la temperatura en ${ }^{\circ} \mathrm{C}$ (siguen el orden de aumento de la escala de tiempo). 
Lentamente la temperatura se ha ido aumentando desde $30^{\circ} \mathrm{C}$ hasta $43^{\circ} \mathrm{C}$ : la diferencia de potencial aumenta solidario con el aumento de la temperatura. Con indicadores triangulares se señalan algunas paradas rutinarias de mantenimiento del sistema: éstas consisten en la reposición del agua utilizada en el electrolizador o la eliminación del agua líquida depositada en los centros donde tiene lugar la catálisis en la batería. Luego de cada parada se observa una rápida llegada a estado de régimen de funcionamiento. Como se mencionó más arriba, después de los primeros dos meses de funcionamiento continuo, la puesta en marcha se hizo a intervalos variables, dependiendo de la disponibilidad de personal en la Base. Durante las 2278 horas de funcionamiento acumuladas hasta el 25 de mayo de 2008 la batería entregó 4,2 kWh. Los resultados son consistentes con las 2000 horas de funcionamiento sin pérdida de potencia informados por Ballard (citado por Haille; 2002) para pilas de combustión con membrana de intercambio iónico.

\section{CONCLUSIONES}

El conjunto desarrollado permite trabajar con un mínimo de componentes periféricos y ser manejado por personal entrenado con capacitación básica para su operación y mantenimiento. La resistencia óhmica del conjunto no difiere de la que se obtendría con seis elementos en serie. Las pendientes de Tafel fueron de $75 \mathrm{mV} /$ década para un elemento simple y de $80 \mathrm{mV} /$ década para la batería, valores coincidentes con los de otros autores.

El comportamiento en diferencia de potencial, corriente y potencia del "stack" de seis elementos bipolares puede considerarse como la suma de seis pilas individuales, lo que indica que no hay pérdidas resistivas significativas resultantes del armado de la batería. Se realizaron estudios de estabilidad de funcionamiento en el tiempo en los cuales el sistema alcanzó 2278 horas de trabajo acumuladas a lo largo de casi 18 meses de instalado, mostrando un comportamiento similar al informado en la literatura.

\section{AGRADECIMIENTOS}

Los autores agradecemos el apoyo económico recibido del convenio entre el Ejército Argentino, Energía Argentina Sociedad Anónima (ENARSA) y Aeropuertos Argentina 2000 (29 de diciembre de 2004) y el subsidio del Ministerio de Defensa PIDDEF 0024/08/CITEDEF. Agradecemos igualmente el apoyo brindado por el Departamento Taller de Prototipos del Instituto de Investigaciones Científicas y Técnicas para la Defensa (CITEDEF) y por el Comando Antártico de Ejército.

\section{REFERENCIAS}

Andreaus, B. y G. Scherer; Proton-conducting polymer membranes in fuel cells-humidification aspects, Solid State Ionics: 168, 311-320 (2004).

Antolini, E., R.R. Passos y E.A. Ticianelli; Effects of the carbon powder characteristics in the cathode gas diffusion layer on the performance of polymer electrolyte fuel cells, Journal of Power Sources: 109, 477- 482, (2002).

Casanova, J., H.J. Fasoli, A.R. Sanguinetti y J.I. Franco; Caracterización electroquímica del electrolito de una pila de combustión, Memorias de las Jornadas SAM-CONAMET-Simposio Materia, San Carlos de Bariloche, 1304-1306 (2003).

Dohle, H., R. Jung, N. Kimiaie, J. Mergel y M. Müller; Interaction between the diffusion layer and the flow field of polymer electrolyte fuel cells: experiments and simulation studies, Journal of Power Sources: 124, 371-384, (2003)

Fasoli, H.J. y J.I. Franco; Pilas de combustión PEM hidrógeno-aire: plan para el desarrollo energético individual, en "Hidrógeno y la nueva energía", editores: Dubois, R., Perazzo, R. y Triaca, W., Academia Nacional de Ciencias Exactas, Físicas y Naturales y Academia Nacional de Ingeniería, 97108 (2004). 
Fasoli, H.J.,J.I. Franco, J.I. y A.R. Sanguinetti; Gestión novedosa en un proyecto sobre energías alternativas con tecnología propia, Actas de la II Jornadas de Vinculación Tecnológica del Consejo Interuniversitario Nacional, Paraná, Argentina, en prensa (2008).

Franco, J.I., H.J. Fasoli, A.R. Sanguinetti, I.P. Grosmann, M.V. D’Atri y C.A. Pavón; Primera batería ("stack") de pilas de combustión PEM a hidrógeno con tecnología propia, Actas del II Congreso Argentino y I Iberoamericano "Hidrógeno y Fuentes Sustentables de Energía" (HYFUSEN), publicación 02-02 (2007).

Giddey, S., F.T Ciachi y S.P. Badwal; Design,assembly and operation of polymer electrolyte membrane fuel cell stacks to 1 kW capacity, Journal of Power Sources: 125, 155-165 (2004).

Haille, S.M.; Fuel cells materials and components, Acta Materialia: 51, 5981-6000 (2003).

Hyun, D. y J. Kim; Study of external humidification method in proton exchange membrane fuel cell, Journal of Power Sources: 126, 98-103 (2004).

Jiao, K. y B. Zhou; Effects of electrode wettabilities on liquid water behaviours in PEM fuel cell cathode, Journal of Power Sources: 175,106 -119 (2008).

Knights, K. Colbow, J. St-Pierre y D. Wilkinson; Aging mechanisms and lifetime PEFC and DMFC, Journal of Power Sources: 127, 127-134 (2004).

Kordesh,K. y G. Simader; Fuel Cells and their applications, VCH Publishers, New York, 12-13, (1996).

Park, J. y X. Li; Effect of flow and temperature distribution on the performance of a PEM fuel cell stack, Journal of Power Sources: 162, 444-459 (2006).

Passos, R. y E.A. Ticianelli; Effects of the operational conditions on the membrane and electrode properties of a polymer electrolyte fuel cell, J. Braz. Chem. Soc.: 3,483-489 (2002).

Qi, Z. y A. Kaufman; Hierarchy carbon paper for the gas diffusion layer of proton exchange membrane fuel cells, Journal of Power Sources: 109, 469 - 476 (2002).

Paganin, V., C. Oliveira, E. Ticianelli, T.Springer y E. González; Modelistic interpretation of the impedance response of a polymer electrolyte fuel cell, Electrochimica Acta: 43, 3761-3766 (1998).

Rodatz, P., F. Büchi, C. Onder y L. Guzzella.; Operacional aspects of a large scale PEFC stack Ander practical conditions, Journal of Power Sources: 128, 208-217 (2004).

Sanguinetti, A.R., H.J. Fasoli y J.I. Franco; “Un procedimiento para la elaboración de electrodos para pilas de combustible y tintas aptas para esa función", CITEFA-EST (cesionarios), P 070103580 , Boletín de Patentes de la Argentina, Año X, Nº 502, 5/11/08, (2007).

Ticianelli, E.A., C.R. Derouin y S.J. Srinivasan; Localization of platinum in low catalyst loading electrodes to attain high power densities in fuel cells, Electroanal. Chem.: 251, 275-286 (1998).

Zhang, L., Ch. Ma y S. Mukerjee; Oxygen permeation studies on alternative proton Exchange membranes designed for elevated temperature operation, Electrochimica Acta: 48 1845-1849 (2001). 